\title{
COEFFICIENTS OF UNIVALENT FUNCTIONS
}

BY PETER L. DUREN

The interplay of geometry and analysis is perhaps the most fascinating aspect of complex function theory. The theory of univalent functions is concerned primarily with such relations between analytic structure and geometric behavior.

A function is said to be univalent (or schlicht) if it never takes the same value twice: $f\left(z_{1}\right) \neq f\left(z_{2}\right)$ if $z_{1} \neq z_{2}$. The present survey will focus upon the class $S$ of functions

$$
f(z)=z+a_{2} z^{2}+a_{3} z^{3}+\cdots
$$

analytic and univalent in the unit disk $|z|<1$. This is the class of all univalent functions normalized by the conditions $f(0)=0$ and $f^{\prime}(0)=1$. We shall concentrate on coefficient problems for the class $S$ and for related classes, with emphasis on recent results and open problems. Most of the methods we shall describe have wide scope and are not restricted to coefficient problems.

The theory of univalent functions is an old but very active field. The last ten or fifteen years have witnessed a number of major advances. In fact, progress has been so rapid that Hayman's 1965 survey [66] of coefficient problems is already rather out of date.

In most general form, the coefficient problem is to determine the region of $\mathbb{C}^{n-1}$ occupied by the points $\left(a_{2}, \ldots, a_{n}\right)$ for all $f \in S$. The deduction of such precise analytic information from the geometric hypothesis of univalence is exceedingly difficult. We shall confine attention to the more special problem of estimating $\left|a_{n}\right|$, the modulus of the $n$th coefficient. Even this problem has never been solved completely.

1. The Bieberbach conjecture. The leading example (aside from the identity) of a function of class $S$ is the Koebe function

$$
k(z)=z(1-z)^{-2}=z+2 z^{2}+3 z^{3}+\cdots,
$$

which maps the unit disk onto the full plane slit along the negative real axis from $-1 / 4$ to $\infty$. The Koebe function and its rotations $k_{\varphi}(z)=e^{-i \varphi} k\left(e^{i \varphi} z\right)$ have long been known (see [55], [63], [120], [38]) to maximize and minimize

An expanded version of an invited address delivered before the Annual Meeting of the Society in San Antonio, Texas, January 23, 1976; received by the editors December 16, 1976.

AMS (MOS) subject classifications (1970). Primary 30-02, 30A34; Secondary 30A36, 30A38, $30 \mathrm{~A} 40$.

Key words and phrases. Univalent functions, coefficient problems, Grunsky inequalities, variational methods.

(1) American Mathematical Society 1977 
various functionals in $S$, such as $\left|f\left(z_{0}\right)\right|$ and $\left|f^{\prime}\left(z_{0}\right)\right|$ at a fixed point $z_{0}$. In 1916, Bieberbach [12] conjectured that they also maximize $\left|a_{n}\right|$, the modulus of the $n$th coefficient.

Bieberbach Conjecture. For each function $f \in S,\left|a_{n}\right| \leqslant n$ for $n=$ $2,3, \ldots$. Strict inequality holds for all $n$ unless $f$ is the Koebe function or one of its rotations.

The Bieberbach conjecture has stood for sixty years and has inspired the development of important new methods in complex analysis. In all likelihood, it has already contributed more to mathematics as a challenging problem than it will ever contribute as a theorem. It has now been verified up to $n=6$, each coefficient being treated by a different method. A review of these proofs will give us an opportunity to describe some of the main techniques of the field.

Bieberbach [11], [12] proved $\left|a_{2}\right| \leqslant 2$ as a corollary to an elementary result known as the area theorem. Closely related to $S$ is the class $\Sigma$ of functions

$$
g(z)=z+b_{0}+b_{1} z^{-1}+b_{2} z^{-2}+\cdots
$$

analytic and univalent in $|z|>1$ except for a simple pole at $\infty$ with residue 1 .

AREA TheOREM. For each $g \in \Sigma, \sum_{n=1}^{\infty} n\left|b_{n}\right|^{2} \leqslant 1$.

PROOF. Since $g$ is univalent, it maps each circle $|z|=r>1$ onto a simple closed curve $C_{r}$ whose interior has positive area expressed (by Green's theorem) as a contour integral:

$$
\begin{aligned}
0<\frac{1}{2 i} \int_{C_{r}} \bar{w} d w & =\frac{1}{2 i} \int_{|z|=r} \overline{g(z)} g^{\prime}(z) d z \\
& =\pi\left\{r^{2}-\sum_{n=1}^{\infty} n\left|b_{n}\right|^{2} r^{-2 n}\right\} .
\end{aligned}
$$

Now let $r \rightarrow 1$ to obtain the result.

In particular, $\left|b_{1}\right| \leqslant 1$. The inequality $\left|a_{2}\right| \leqslant 2$ follows by considering $g(z)=\left\{f\left(1 / z^{2}\right)\right\}^{-1 / 2}$, which belongs to $\Sigma$. The area theorem is due to Gronwall $[60]$.

In 1923, Loewner [92] proved $\left|a_{3}\right| \leqslant 3$, using what is essentially a parametric representation of the slit mappings. These are the functions which map the disk onto the full plane slit along some Jordan arc terminating at $\infty$. The slit mappings are dense in $S$ in the topology of uniform convergence on compact subsets of the disk. Loewner proved they can be generated (along with certain other univalent functions) by a differential equation of prescribed form. This leads to an explicit integral representation of $a_{3}$, from which the estimate follows.

The problem of the fourth coefficient remained open until 1955, when Garabedian and Schiffer [46] used a variational method to show that $\left|a_{4}\right| \leqslant 4$. Their proof was extremely complicated. Schiffer [134], [135], [136] had developed a calculus of variations for the class $S$ and had used it to show that each function in $S$ which maximizes $\left|a_{n}\right|$ must map the disk onto the plane slit 
along a system of analytic arcs satisfying a certain differential equation, the trajectories of a certain quadratic differential. The main difficulty in applying the variational method is the global analysis of the trajectory structure. The general coefficient theorem as developed by Jenkins [75], [76], [77], [78] complements the variational method in a remarkable way and automatically gives an inequality involving the coefficients of the extremal function, but from this it is of ten difficult to deduce the desired inequality.

Five years after the Garabedian-Schiffer proof, Charzynski and Schiffer [23] astonished everyone with the discovery of an elementary (and simple!) proof of $\left|a_{4}\right| \leqslant 4$. It was based on the Grunsky inequalities [61], which can be derived by a slight generalization of the method used to prove the area theorem. One statement is as follows. For $g \in \Sigma$, let

$$
\log \frac{g(z)-g(\zeta)}{z-\zeta}=\sum_{n=1}^{\infty} \sum_{k=1}^{\infty} c_{n k} z^{-n} \xi^{-k}, \quad|z|>1,|\xi|>1 .
$$

The coefficients $c_{n k}$ are polynomials in the coefficients $b_{n}$ of $g$.

GrunsKy INEQUALITIES. For each integer $N$ and for all complex numbers $\lambda_{1}, \lambda_{2}, \ldots, \lambda_{N}$,

$$
\left|\sum_{n=1}^{N} \sum_{k=1}^{N} c_{n k} \lambda_{n} \lambda_{k}\right| \leqslant \sum_{n=1}^{N} \frac{1}{n}\left|\lambda_{n}\right|^{2} .
$$

For a technical reason, the Grunsky inequalities seem to apply to the Bieberbach conjecture more readily for even indices than for odd. In 1968, Pederson [110] and Ozawa [108], [109] used them to prove $\left|a_{6}\right| \leqslant 6$. Only several years later did Pederson and Schiffer [112] succeed in proving $\left|a_{5}\right| \leqslant 5$. They applied the Garabedian-Schiffer inequalities, a generalization of the Grunsky inequalities which Garabedian and Schiffer [48] had derived by a variational method. These inequalities involve auxiliary parameters which can be chosen to optimize the estimates.

The Bieberbach conjecture has been established for various subclasses of $S$. For example, it is true for starlike functions (that is, for functions whose range is starlike with respect to the origin) [91], [106], for "close-to-convex" functions [80], [122], for functions with real coefficients [28], [127], [145], and even for functions whose first $[n / 2]$ coefficients are real [58], [107], [139]. With the aid of a computer, D. Horowitz [69] recently verified it for polynomials in $S$ of degree up to 27.

For the full class $S$, Littlewood [88] showed that $\left|a_{n}\right|<e n$ for all $n$, where $e$ is the base of natural logarithms. His proof involves the crude estimation of the Cauchy integral formula for $a_{n}$ :

$$
\left|a_{n}\right| \leqslant r^{-n} M_{1}(r, f), \quad 0<r<1,
$$

where

$$
M_{p}(r, f)=\left\{\frac{1}{2 \pi} \int_{0}^{2 \pi}\left|f\left(r e^{i \theta}\right)\right|^{p} d \theta\right\}^{1 / p}, \quad 0<p<\infty
$$


Littlewood used an elegant geometric argument to prove $M_{1}(r, f) \leqslant$ $r(1-r)^{-1}$, whereupon the choice $r=1-1 / n$ leads to the conclusion. The crude estimate $\left|a_{n}\right| \leqslant r^{-n} M_{1}(r, f)$ can lead to nothing better than $\left|a_{n}\right|<$ $e n / 2$, since the Koebe function has integral mean $M_{1}(r, k)=r\left(1-r^{2}\right)^{-1}$. Bazilevič [9] improved Littlewood's estimate for $M_{1}(r, f)$ to show

$$
\left|a_{n}\right|<e n / 2+1.51, \quad n=2,3, \ldots
$$

The sharp bound for $M_{1}(r, f)$ remained undetermined until a few years ago, when Baernstein [7] was able to prove $M_{p}(r, f) \leqslant M_{p}(r, k), 0<p<\infty$, for all $f \in S$. In particular, this completes the proof that $\left|a_{n}\right|<e n / 2$.

The first penetration of the $e / 2$-barrier came in 1965, when Milin [98], [102] proved $\left|a_{n}\right|<1.243 n$. More recently, FitzGerald [44] showed

$$
\left|a_{n}\right|<(7 / 6)^{1 / 2} n<1.081 n .
$$

Later we shall describe the methods of Milin and FitzGerald, which are somewhat similar. Horowitz [68] has refined FitzGerald's method to obtain the best estimate now known:

$$
\left|a_{n}\right|<(209 / 140)^{1 / 6} n<1.0691 n .
$$

In connection with Baernstein's theorem, it should be remarked that the corresponding problem for derivatives is still unsolved. The inequality $M_{p}\left(r, f^{\prime}\right) \leqslant M_{p}\left(r, k^{\prime}\right)$ is false for $p<1 / 3$, since $k^{\prime} \in H^{p}$ for all $p<1 / 3$ and it is known [93], [38] that there exist univalent functions $f$ with $f^{\prime} \notin H^{p}$ for all $p>0$. It follows easily from Baernstein's theorem that $M_{p}\left(r, f^{\prime}\right)=$ $O\left((1-r)^{1 / p-3}\right)$ for $f \in S$ and $p>1 / 2$. (For $p \geqslant 1$ this is a consequence of Littlewood's estimate of $M_{1}(r, f)$.) Feng and MacGregor [43] recently extended this (best possible) asymptotic result to $p>2 / 5$. For close-to-convex functions $f$, Cluni and I [31], [26] proved $M_{1}\left(r, f^{\prime}\right) \leqslant M_{1}\left(r, k^{\prime}\right)$, and MacGregor [95] generalized this to all $p \geqslant 1$. It is a reasonable conjecture that this inequality $M_{p}\left(r, f^{\prime}\right) \leqslant M_{p}\left(r, k^{\prime}\right)$ extends to the full class $S$ for all $p>1 / 3$.

2. Odd univalent functions. Another problem closely related to the Bieberbach conjecture is to find the sharp estimates for the coefficients of odd univalent functions. The most general odd function of class $S$ has the form

$$
n(z)=\left[f\left(z^{2}\right)\right]^{1 / 2}=z+c_{3} z^{3}+c_{5} z^{5}+\cdots, \quad f \in S .
$$

Littlewood and Paley [90] showed in 1932 that $\left|c_{n}\right| \leqslant A$ for all $n$, where $A$ is an absolute constant (their method gives $A<14$ ), and they added the footnote, "No doubt the true bound is given by $A=1$."

This last remark has become known as the Littlewood-Paley conjecture. It easily implies the Bieberbach conjecture, as the following argument shows. Compare coefficients of $z^{2 n}$ in the equation $f\left(z^{2}\right)=[h(z)]^{2}$ to derive the relation

$$
a_{n}=c_{1} c_{2 n-1}+c_{3} c_{2 n-3}+\cdots+c_{2 n-1} c_{1}, \quad c_{1}=1 .
$$


Since there are $n$ terms on the right-hand side, the uniform bound $\left|c_{k}\right| \leqslant 1$ would imply $\left|a_{n}\right| \leqslant n$. In fact, an application of the Cauchy-Schwarz inequality shows that the following conjecture, proposed by Robertson [123] in 1936, also implies the Bieberbach conjecture.

Robertson CONJecture. For each odd function $h(z)=z+c_{3} z^{3}+c_{5} z^{5}$ $+\cdots$ of class $S$,

$$
1+\left|c_{3}\right|^{2}+\cdots+\left|c_{2 n-1}\right|^{2} \leqslant n, \quad n=2,3, \ldots
$$

For $n=2$ this inequality is equivalent to $\left|a_{2}\right| \leqslant 2$. Robertson [123] used Loewner's method to prove it for $n=3$. In 1970, Friedland [45] applied the Grunsky inequalities to extend it to $n=4$, but for larger $n$ it remains open. It seems to be open even for functions with real coefficients.

The Littlewood-Paley conjecture is true for starlike functions [121], [50] but false in general. As early as 1933, Fekete and Szegö [42] applied the Loewner method to obtain the sharp inequality

$$
\left|c_{5}\right| \leqslant 1 / 2+e^{-2 / 3}=1.013 \ldots
$$

In fact, they obtained the sharp bound

$$
\left|a_{3}-\alpha a_{2}^{2}\right| \leqslant 1+2 e^{-2 \alpha /(1-\alpha)}, \quad 0 \leqslant \alpha \leqslant 1,
$$

which gives the result because $c_{5}=\left(a_{3}-a_{2}^{2} / 4\right) / 2$.

Schaeffer and Spencer [131] later produced an elementary construction, for each odd integer $n \geqslant 5$, of an odd function of class $S$ with real coefficients and with $\left|c_{n}\right|>1$. They also found, using a variational method, that the Fekete-Szegö bound for $\left|c_{5}\right|$ is attained for a function with real coefficients. Quite recently, Leeman [85] has shown that for odd functions in $S$ with real coefficients the sharp bound for $\left|c_{7}\right|$ is 1090/1083. The appearance of a rational bound was totally unexpected. The upper and lower bounds for $c_{7}$ are symmetric, since the passage from $h(z)$ to $-i h(i z)$ preserves real coefficients and reverses the sign of $c_{n}$ for each $n \equiv 3(\bmod 4)$.

The best known estimate of the Littlewood-Paley constant is due to Milin [99], [102]: $A \leqslant 1.17$. This represents an improvement on earlier results of $\mathrm{V}$. I. Levin [87] and Kung Sun [83].

3. Asymptotic results. For each given function $f \in S$, the Bieberbach conjecture is eventually true: Hayman [62] showed in 1955 that $\left|a_{n}\right| / n$ always tends to a limit less than one unless $f$ is a rotation of the Koebe function. Actually, Hayman's full result involves multivalent functions and is much more general, but we state it in specialized form.

Hayman Regularity Theorem. For each fixed $f \in S$,

$$
\lim _{n \rightarrow \infty}\left|a_{n}\right| / n=\alpha \leqslant 1,
$$


with equality if and only if $f$ is a rotation of the Koebe function.

This theorem does not prove the Bieberbach conjecture for large $n$ because $\alpha$ depends on $f$ and the convergence is not uniform, even if $\alpha$ is prescribed. In fact, Shirokov [144] recently showed that $\left|a_{n}\right| / n$ may tend to any given $\alpha$ arbitrarily slowly.

Hayman's proof proceeds in two main steps. The first step is to show that for each $f \in S$,

$$
\lim _{r \rightarrow 1}(1-r)^{2} M_{\infty}(r, f)=\alpha \leqslant 1,
$$

where $M_{\infty}(r, f)$ denotes the maximum of $|f(z)|$ on the circle $|z|=r$. An easy compactness argument then proves that $f$ has a direction $e^{i \theta}$ of maximal growth:

$$
\lim _{r \rightarrow 1}(1-r)^{2}\left|f\left(r e^{i \theta}\right)\right|=\alpha .
$$

The second and more difficult step is to deduce that $\left|a_{n}\right| / n$ tends to this same number $\alpha$. For $\alpha>0$, Milin [101], [102] has found an alternate approach to this latter step which suggests viewing it as a Tauberian theorem. Recently, I applied Tauberian remainder theorems to obtain quantitative versions of Hayman's theorem [34], [35].

Hayman's theorem appears to be persuasive evidence in favor of the Bieberbach conjecture until it is compared with Hayman's parallel result for odd functions

$$
h(z)=\left[f\left(z^{2}\right)\right]^{1 / 2}=z+c_{3} z^{3}+c_{5} z^{5}+\cdots, \quad f \in S .
$$

If $f$ has Hayman number $\alpha$, then $\left|c_{2 n+1}\right| \rightarrow \sqrt{\alpha} \leqslant 1$, despite the failure of the Littlewood-Paley conjecture.

The Bieberbach conjecture is known to be true for functions with sufficiently small second coefficient. Aharonov [1], [3] and Il'ina [73] adapted Milin's method to show that if $\left|a_{2}\right|<1.05$, then $\left|a_{n}\right|<n$ for all $n$. More recently, Ehrig [41] applied an inequality of FitzGerald to improve the constant to 1.15, and Bishouty [14] used a closely related technique to extend it to 1.55. (In unpublished work, Bishouty has since pushed it up to 1.61.) Ehrig [40], [41] also showed that if $f \in S$ and $\left|a_{2}\right| \leqslant C<1.709$, then $\left|a_{n}\right|<n$ for all $n \geqslant N$, where $N$ depends only on $C$ and not (as in Hayman's theorem) on $f$.

The Hayman number $\alpha$ can be estimated in terms of the bound $C$ on $\left|a_{2}\right|$. As a corollary to Jenkins' [74] sharp estimate of $|f(z)|$ for functions $f \in S$ with fixed $a_{2}$, Hayman [62] gave the sharp bound

$$
\alpha \leqslant 4 \lambda^{2} e^{2-4 \lambda}, \quad \lambda=\left\{2-(2-C)^{1 / 2}\right\}^{-1} .
$$

Hayman [64] also proved that $A_{n} / n$ tends to a limit, where $A_{n}$ is the maximum of $\left|a_{n}\right|$ for all $f \in S$. It is still an open question whether this limit is equal to 1 . 
Asymptotic Bieberbach Conjecture. $\lim _{n \rightarrow \infty} A_{n} / n=1$, where $A_{n}=$ $\max _{f \in S}\left|a_{n}\right|$.

Yet another unsettled conjecture was proposed by Littlewood [88] in 1925.

LitTLEWOOD CONJECTURE. If $f \in S$ and $f(z) \neq w$ for some complex number $w$, then $\left|a_{n}\right| \leqslant 4|w| n, n=2,3, \ldots$

By a classical theorem of Koebe, any omitted value $w$ must have modulus $|w| \geqslant 1 / 4$. Thus the Littlewood conjecture is an immediate consequence of the Bieberbach conjecture. Nehari [103] showed that even the asymptotic Bieberbach conjecture implies the Littlewood conjecture.

4. Exponentiation of the Grunsky inequalities. Roughly speaking, the Grunsky inequalities give detailed information about the coefficients of the logarithm of a univalent function. Milin's leading idea is to transfer this to information about the coefficients of the function itself by a process of exponentiation, applying some inequalities due to Lebedev and himself. These inequalities concern general power series and have nothing to do per se with univalent functions. Let $\varphi(z)=\sum_{k=1}^{\infty} \alpha_{k} z^{k}$ be an arbitrary power series with a positive radius of convergence, normalized by $\varphi(0)=0$. Let

$$
e^{\varphi(z)}=\sum_{k=0}^{\infty} \beta_{k} z^{k}, \quad \beta_{0}=1,
$$

denote the exponentiated power series.

LEBEDEV-MILIN INEQUALITIES.

$$
\begin{aligned}
\sum_{k=0}^{\infty}\left|\beta_{k}\right|^{2} & \leqslant \exp \left\{\sum_{k=1}^{\infty} k\left|\alpha_{k}\right|^{2}\right\}, \\
\frac{1}{n+1} \sum_{k=0}^{n}\left|\beta_{k}\right|^{2} & \leqslant \exp \left\{\frac{1}{n+1} \sum_{m=1}^{n} \sum_{k=1}^{m}\left(k\left|\alpha_{k}\right|^{2}-1 / k\right)\right\}, \\
\left|\beta_{n}\right|^{2} & \leqslant \exp \left\{\sum_{k=1}^{n}\left(k\left|\alpha_{k}\right|^{2}-1 / k\right)\right\} .
\end{aligned}
$$

All three inequalities are sharp. For proofs, see [102], [2], [120], [38].

The logarithmic coefficients of a function $f \in S$ are the numbers $\gamma_{n}$ defined by

$$
\log \frac{f(z)}{z}=2 \sum_{n=1}^{\infty} \gamma_{n} z^{n}, \quad|z|<1 .
$$

If $f$ is the Koebe function, then $\gamma_{n}=1 / n$. A remarkable result of Bazilevič [10], [102] asserts that

$$
\sum_{n=1}^{\infty} n\left|\gamma_{n}-\frac{1}{n}\right|^{2} \leqslant \frac{1}{2} \log \frac{1}{\alpha}
$$


for functions $f \in S$ with positive Hayman number $\alpha$, rotated so that 1 is the direction of maximal growth. This inequality says that if $\alpha$ is near 1 , then $f$ is "near" the Koebe function. For starlike functions $f$, it is not hard to show that $\left|\gamma_{n}\right| \leqslant 1 / n$ for all $n$. This is false in general, even in order of magnitude [116], [118], [59]. Nevertheless, Milin [99], [102] used the Grunsky inequalities to obtain the following result.

Milin Lemma. For every function $f \in S$,

$$
\sum_{k=1}^{n} k\left|\gamma_{k}\right|^{2} \leqslant \sum_{k=1}^{n} \frac{1}{k}+\delta
$$

where $\delta<0.312$.

Corollary. The coefficients of odd univalent functions satisfy $\left|c_{n}\right| \leqslant e^{\delta / 2}$ $<1.17, n=3,5, \ldots$.

The corollary is derived from Milin's lemma by applying the third LebedevMilin inequality. Since the Littlewood-Paley conjecture is false, the constant $\delta$ cannot be reduced to zero. Milin has offered the following conjecture, however, which asserts that $\delta=0$ in an average sense.

Milin Conjecture. For each $f \in S$,

$$
\sum_{m=1}^{n} \sum_{k=1}^{m}\left(k\left|\gamma_{k}\right|^{2}-\frac{1}{k}\right) \leqslant 0, \quad n=1,2, \ldots
$$

An application of the second Lebedev-Milin inequality shows that the Milin conjecture implies the Robertson conjecture, and so implies the Bieberbach conjecture. Grinšpan [59] has verified the Milin conjecture up to $n=3$.

Closely related to the Grunsky inequalities is a system of inequalities due to Goluzin [52], [55], [120], [38], restricting the values a univalent function can take at prescribed points. Although Goluzin originally derived these inequalities by Loewner's method, they are easily obtained from the Grunsky inequalities, and vice-versa.

GoluZIN INEQUALITIES. For each $f \in S$, for distinct points $z_{n}\left(0<\left|z_{n}\right|<1\right)$, and for arbitrary complex numbers $\lambda_{n}$,

$$
\begin{aligned}
\left|\sum_{n=1}^{N} \sum_{k=1}^{N} \lambda_{n} \lambda_{k} \log \frac{z_{n} z_{k}\left[f\left(z_{n}\right)-f\left(z_{k}\right)\right]}{f\left(z_{n}\right) f\left(z_{k}\right)\left(z_{n}-z_{k}\right)}\right| \\
\leqslant \sum_{n=1}^{N} \sum_{k=1}^{N} \lambda_{n} \overline{\lambda_{k}} \log \frac{1}{1-z_{n} \overline{z_{k}}} .
\end{aligned}
$$

(For $k=n$, the difference quotient is interpreted as a derivative.)

The Goluzin inequalities are the point of departure for FitzGerald's proof that $\left|a_{n}\right|<(7 / 6)^{1 / 2} n$. The first step is to "exponentiate" the Goluzin inequalities to get rid of the logarithms. The resulting inequalities are 


$$
\left.\left.\left|\sum_{n=1}^{N} \lambda_{n}\right| \frac{f\left(z_{n}\right)}{z_{n}}\right|^{2}\left|\leqslant \sum_{n=1}^{N} \sum_{k=1}^{N} \lambda_{n} \overline{\lambda_{k}}\right| \frac{f\left(z_{n}\right)-f\left(z_{k}\right)}{\left(z_{n}-z_{k}\right)\left(1-z_{n} \overline{z_{k}}\right)}\right|^{2} .
$$

Although FitzGerald derived them $a b$ initio by recourse to the Loewner method, they can be deduced from the Goluzin inequalities with the aid of some purely algebraic results of Schur [142] concerning positive-definite quadratic forms (see [120], [38]). The second step is to make a suitable choice of the parameters $\lambda_{n}$ and $z_{n}$, and to perform certain integrations to obtain

$$
\left|a_{n}\right|^{4} \leqslant \sum_{k=1}^{n} k\left|a_{k}\right|^{2}+\sum_{k=n+1}^{2 n-1}(2 n-k)\left|a_{k}\right|^{2} \text {. }
$$

From this it is easy to conclude that $\left|a_{n}\right|<(7 / 6)^{1 / 2} n$.

5. Successive coefficients. Another problem which has attracted considerable attention is to estimate $d_{n}=|| a_{n+1}|-| a_{n}||, n=2,3, \ldots$, the difference of the moduli of successive coefficients of functions in $S$. Goluzin [51] showed $d_{n}=O\left(n^{1 / 4} \log n\right)$, and Biernacki [13] improved this to $d_{n}=O\left((\log n)^{3 / 2}\right)$. Then in 1963 Hayman [65] settled the order of growth problem by showing that $d_{n} \leqslant A$, where $A$ is an absolute constant. Milin [100], [102] found an alternate proof, simpler than Hayman's, which led to the bound $d_{n}<9$. Il'ina [72] then refined Milin's method to obtain the best bound now known: $d_{n}<4.17$. (A trivial modification of the proof, appealing to the FitzGeraldHorowitz bound for $\left|a_{n}\right|$ instead of to Milin's, improves slightly on the estimate 4.26 given in Il'ina's paper.) See also [102], [120], [38]. The bound cannot be reduced to 1 because for each $n \geqslant 4$ there are odd univalent functions with $d_{n}>1$. Goluzin [51], [53] and Jenkins [78] found the sharp bounds

$$
-1 \leqslant\left|a_{3}\right|-\left|a_{2}\right| \leqslant 3 / 4+e^{-\lambda}\left(2 e^{-\lambda}-1\right)=1.029 \ldots,
$$

where $4 \lambda e^{-\lambda}=1,0<\lambda<1$. (Actually, an algebraic slip led Goluzin to a spurious solution, but his proof by Loewner's method can be salvaged; see [38].)

The corresponding problem for odd univalent functions, to find the best asymptotic estimate for $\delta_{n}=|| c_{2 n+1}\left|-c_{2 n-1}\right| \mid$, is still unsolved. It is known that $\delta_{n} \rightarrow 0$, and Goluzin [54] showed $\delta_{n}=O\left(n^{-1 / 4} \log n\right)$. The best estimate currently known is $\delta_{n}=O\left(n^{1-\sqrt{2}}\right)$, due to Lucas [94]. The truth may well be $\delta_{n}=O\left(n^{-1 / 2}\right)$. The example $\left[k\left(z^{4}\right)\right]^{1 / 4}$, where $k$ is the Koebe function, shows nothing better is possible. For odd functions $h(z)=\left[f\left(z^{2}\right)\right]^{1 / 2}$ arising as square-root transforms of functions $f \in S$ with positive Hayman number $\alpha$, Milin [102], [38] has shown

$$
\delta_{n}<\alpha^{-1 / 4} n^{-1 / 2}, \quad n=1,2, \ldots
$$

For functions $h(z)=\left[f\left(z^{4}\right)\right]^{1 / 4}$ with four-fold symmetry, Levin [86] showed long ago that $c_{4 n+1}=O\left(n^{-1 / 2} \log n\right)$. It seems likely that the logarithmic factor 
is superfluous, but even this is an open question.

6. Subordination. A function $g(z)=b_{1} z+b_{2} z^{2}+\cdots$ analytic in $|z|<1$ is said to be subordinate to a function $f \in S$ (written $g<f$ ) if the range of $g$ is contained in that of $f$. The Schwarz lemma applied to $f^{-1}(g(z))$ then shows that $g$ has the form $g(z)=f(\omega(z))$ for some analytic function $\omega$ with $|\omega(z)| \leqslant .|z|$. The definition is extended to general analytic functions $f(z)$ $=a_{1} z+a_{2} z^{2}+\cdots$, univalent or not, by requiring that $g$ have this form. A classical theorem of Littlewood [88], [32] asserts that subordination implies mean domination: if $g<f$, then $M_{p}(r, g) \leqslant M_{p}(r, f)$ for $0<r<1$ and $0<p<\infty$. Using this result, Rogosinski [128] deduced the inequality

$$
\sum_{n=1}^{N}\left|b_{n}\right|^{2} \leqslant \sum_{n=1}^{N}\left|a_{n}\right|^{2} \quad N=1,2, \ldots
$$

This inequality is remarkable in that it holds only for squares and is false for all exponents $p \neq 2$. Even if $f$ is univalent, the subordination condition $g<f$ does not imply $\left|b_{n}\right| \leqslant\left|a_{n}\right|$. For example, $z^{2}<z$. However, Littlewood and Rogosinski advanced the following conjecture, known as the generalized Bieberbach conjecture or the Rogosinski conjecture.

Rogosinski Conjecture. If $g \prec f \in S$, then $\left|b_{n}\right| \leqslant n, n=1,2, \ldots$

For $n=1$, the Rogosinski conjecture is contained in the Schwarz lemma. Littlewood [88] proved it for $n=2$. Rogosinski [129] proved it for all $n$ under the additional assumptions that $f$ is starlike or has real coefficients. Robertson [124] verified it for close-to-convex functions. More recently, Robertson [126] observed that the Rogosinski conjecture is implied for each $n$ by the Robertson conjecture; see also [37]. This extends the validity of the Rogosinski conjecture up to $n=4$. For general $n$, the best known estimate is $\left|b_{n}\right|<e n / 2$, obtained by combining Littlewood's theorem with Baernstein's:

$$
M_{1}(r, g) \leqslant M_{1}(r, f) \leqslant M_{1}(r, k)=r\left(1-r^{2}\right)^{-1} .
$$

A recent conjecture of Sheil-Small [143] lies between the Robertson and Rogosinski conjectures. The convolution (or Hadamard product) of two power series $f(z)=\sum a_{n} z^{n}$ and $g(z)=\sum b_{n} z^{n}$ is defined as $h=f * g$, where $h(z)=\sum a_{n} b_{n} z^{n}$.

Sheil-Small Conjecture. For each $f \in S$ and for each polynomial $P$ of degree $n,\|P * f\|_{\infty} \leqslant n\|P\|_{\infty}$.

Here \|\|$_{\infty}$ denotes the maximum modulus in $|z| \leqslant 1$. The choice $P(z)=z^{n}$ shows that the Sheil-Small conjecture implies the Bieberbach conjecture. Sheil-Small proved that it also implies the Rogosinski conjecture, and that it is implied by the Robertson conjecture.

In connection with convolutions, it should be mentioned that Ruscheweyh and Sheil-Small [130] recently established a well-known conjecture of Pólya and Schoenberg [114] by showing that $f * g$ is convex (i.e., is univalent and has 
convex range) whenever $f$ and $g$ are convex.

By way of summary, it is interesting to note that seven of the coefficient conjectures we have mentioned are related by a chain of implications:

$$
\begin{aligned}
\text { Milin Conjecture } & \Rightarrow \text { Robertson Conjecture } \\
\Rightarrow \text { Sheil-SMall Conjecture } & \Rightarrow \text { Rogosinski Conjecture } \\
\Rightarrow \text { Bieberbach Conjecture } & \Rightarrow \text { Asymptotic Bieberbach Conjecture } \\
& \Rightarrow \text { LitTlewood Conjecture. }
\end{aligned}
$$

All are open.

7. Extreme points. At first glance it appears that the methods of functional analysis, though useful in other branches of function theory, can shed no light on extremal problems for univalent functions. The space $S$ is far from linear, since univalence need not be preserved under addition. In fact, A. W. Goodman [57] has exhibited a pair of functions in $S$ whose sum has infinite valence.

Nevertheless, it is fruitful to view $S$ as a subset of the linear space $A$ of all analytic functions in the unit disk. Under the topology of uniform convergence on compact subsets of the disk, $A$ is a locally convex space and $S$ is a compact subset. The Krein-Milman theorem [29] therefore asserts that $S$ is contained in the closed convex hull of its extreme points. This reduces the solution of any linear extremal problem in $S$ to its solution over the set of extreme points of $S$. By definition, a linear extremal problem consists of maximizing $\operatorname{Re}\{L(f)\}$ over $S$, where $L$ is some continuous linear functional on $A$. For example, the Bieberbach problem of maximizing $\left|a_{n}\right|$ is equivalent to the linear problem of maximizing $\operatorname{Re}\left\{a_{n}\right\}$, since $S$ is invariant under rotations.

Thus it is important to identify the extreme points of $S$. This is an open problem, but Brickman [20] showed by an elementary argument that each extreme point of $S$ maps the disk onto the complement of a continuous curve tending to $\infty$ with increasing modulus. The Koebe function and its rotations are certainly extreme points, but $S$ has other extreme points as well. In fact, it is known [21] that the closed convex hull of the Koebe function and its rotations contains the starlike functions, but not all of $S$. The existence of other extreme points may also be inferred from the fact [141, p. 84] that some linear extremal problems over $S$ admit no rotations of the Koebe function as solutions. A remarkable result of Hengartner and Schober [67] asserts that for each extreme point $f$, both $f(z) / z$ and $\log \{f(z) / z\}$ are univalent.

Extreme point theory has been applied with success to a number of problems involving special classes of functions, univalent and otherwise. For instance, Brannan, Clunie, and Kirwan [19] used it in their recent solution of the coefficient problem for functions of bounded boundary rotation. (See also Aharonov and Friedland [4], [5].)

8. Qualitative results. One approach to the Bieberbach conjecture is to consider the extremal problem of maximizing $\operatorname{Re}\left\{a_{n}\right\}$ in the class $S$ and to look 
for properties of the extremal functions (whose existence is assured by the compactness of $S$ ) which will eliminate all but the Koebe function and its rotations. This is the general strategy of the calculus of variations, developed for application to univalent function theory by Schiffer in the late 1930's. The idea of a variational method is to discover properties of an extremal function by comparing it with "nearby" functions in $S$, much in the spirit of elementary calculus. The typical result is the qualitative information that the extremal function maps the disk onto the complement of a system of analytic arcs satisfying a differential equation of prescribed form. This is analogous to the Euler equation in the classical calculus of variations.

Although the present discussion is confined to the coefficient problem, most of the results extend directly to an arbitrary linear extremal problem. Variational methods also apply easily to nonlinear problems.

Even elementary variational methods lead to some interesting results. A function in $S$ can be varied by composing it with a self-mapping of the disk close to the identity. This simple device reveals that the coefficients of each function maximizing $\operatorname{Re}\left\{a_{n}\right\}$ for fixed $n \geqslant 2$ must satisfy

$$
(n+1) a_{n+1}-2 a_{2} a_{n}-(n-1) \overline{a_{n-1}}=0 .
$$

This is due to Marty [96] and is known as the Marty relation. (Observe that the Koebe function is still in contention.) In the same paper, Marty used another elementary variation to show that the range of an extremal function must be dense in the complex plane.

One of the most powerful variational methods is Schiffer's method of boundary variation [134], [135], [137], [138], [71], [141], [38]. Very briefly, the idea is to compose a univalent function $f$ with a certain family of functions univalent on the range of $f$ and close to the identity. Applied to the coefficient problem $\max \operatorname{Re}\left\{a_{n}\right\}$, the method yields the information that the omitted set $\Gamma$ of each extremal function $f \in S$ is a system of analytic arcs $w=w(t)$ satisfying

$$
\frac{1}{w^{2}} P_{n}\left(\frac{1}{w}\right)\left(\frac{d w}{d t}\right)^{2}<0
$$

where $P_{n}$ is a monic polynomial of degree $n-1$ generated by

$$
\frac{\zeta[f(z)]^{2}}{1-\zeta f(z)}=\sum_{\nu=2}^{\infty} P_{\nu}(\zeta) z^{\nu}
$$

The relation $(*)$ determines a direction field for $\Gamma$. A delicate argument shows that $P_{n}(1 / w)$ cannot vanish on $\Gamma$, so that $\Gamma$ has no finite branch-points or corners. In other words, $\Gamma$ consists of a finite number of analytic arcs joined only at $\infty$.

In fact, $\Gamma$ is a single arc. This step was originally taken [132], [140], [113] by variational methods, and with considerable difficulty. Brickman and Wilken [22] recently found a strikingly simply proof, based on Brickman's partial 
description of the extreme points. Brickman's argument [20] actually shows that any function $f \in S$ which omits two values of equal modulus must be a proper convex combination of two functions in $S$, both of which omit open sets. Since such functions cannot be extremal (Marty's result), it follows at once that $f$ is not extremal if it omits a pair of points of equal modulus. Hence $\Gamma$ is a single analytic arc extending to $\infty$ with increasing modulus.

A further argument based on $(*)$ reveals that $\Gamma$ has an even stronger monotonicity property. The tangent vector at each point $w$ of $\Gamma$ makes an angle no larger than $\pi / 4$ with the radius vector from 0 to $w$. It is also known that $\Gamma$ has an asymptotic direction at $\infty$. A proof of the Bieberbach conjecture would consist of showing that $\Gamma$ is a linear ray.

A differential equation for the extremal function $f$ itself can be obtained by introducing the parametrization $w=f\left(e^{i t}\right)$ into $(*)$ and applying the Schwarz reflection principle. The result is

$$
\left\{\frac{z f^{\prime}(z)}{f(z)}\right\}^{2} P_{n}\left(\frac{1}{f(z)}\right)=R_{n}(z)
$$

where $R_{n}$ is the rational function

$$
R_{n}(z)=(n-1) a_{n}+\sum_{\nu=1}^{n-1}\left(\nu a_{\nu} z^{\nu-n}+\nu \overline{a_{\nu}} z^{n-\nu}\right) .
$$

This is known as the Schiffer differential equation. It can also be derived, together with $(*)$, by the method of interior variation [136], [132], [39], [138], [71], [38], which uses potential theory to obtain a variation for Green's function of the range domain. Still another derivation comes from a variational method due to Goluzin [55], [119], [120], [38].

The Schiffer equation is a curious functional-differential equation which each extremal function must satisfy. The Koebe function is a solution, but other solutions (not extremal functions) have long been known; see [6]. An expansion of the left-hand side into power series and a comparison of the coefficients of $z$ gives again the Marty relation. Thus the Schiffer differential equation may be viewed as an infinite sequence of "higher Marty relations".

9. Local results. In the early 1960's Schiffer and I [39] extended the method of interior variation by developing a formula for the second variation of a function in $S$, analogous to the second derivative in ordinary calculus. The resulting formula for the second variation of $\operatorname{Re}\left\{a_{n}\right\}$ is complicated, but it simplifies when applied at a critical point (i.e., to a function satisfying the Schiffer differential equation). For the Koebe function, it reduces essentially to a certain quadratic form which is positive-definite if and only if the Koebe function is a local maximum for the $n$th coefficient, in the sense that the second variation of $\operatorname{Re}\left\{a_{n}\right\}$ is always negative there. We verified [39] the positive-definiteness directly for $n \leqslant 9$, and Bombieri [16] later verified it in general, for all $n$.

The question remained whether the Koebe function is a local maximum for 
the $n$th coefficient in the strong sense that $\operatorname{Re}\left\{a_{n}\right\} \leqslant n$ whenever $\left|a_{2}-2\right|<\varepsilon_{n}$, for some $\varepsilon_{n}>0$. Garabedian, Ross, and Schiffer [49] used a perturbation of the Grunsky inequalities to prove this for all even $n$. Bombieri [15], [17] combined the theory of the second variation with Loewner's method to obtain a proof for all $n$, although he presented the details only for odd $n$. Finally, Garabedian and Schiffer [48] developed their generalized Grunsky inequalities (mentioned in \$1) and used them to give an independent proof of the local theorem for all odd $n$. Pederson [111] established the equivalence of various topologies near the Koebe function.

10. Coefficient problem for the class $\Sigma$. The area theorem (\$1) shows that the coefficients of functions

$$
g(z)=z+b_{1} z^{-1}+b_{2} z^{-2}+\cdots
$$

of class $\Sigma$ satisfy $\left|b_{n}\right| \leqslant n^{-1 / 2}$. This is sharp only for $n=1$, in which case the extremal functions (normalized so that $b_{0}=0$ ) have the form

$$
g(z)=z+b_{1} z^{-1}, \quad\left|b_{1}\right|=1,
$$

and map $|z|>1$ onto the complement of a line segment. In 1938, Schiffer [133] used a variational method to establish the sharp inequality $\left|b_{2}\right| \leqslant 2 / 3$, with equality only for the function

$$
g(z)=\left\{k\left(z^{-3}\right)\right\}^{-1 / 3}=z-2 z^{-2} / 3+\cdots
$$

and its rotations, where $k$ is the Koebe function.

This evidence suggested the conjecture $\left|b_{n}\right| \leqslant 2 /(n+1)$, with equality for

$$
\left\{k\left(z^{-n-1}\right)\right\}^{-1 /(n+1)}=z-2 z^{-n} /(n+1)+\cdots,
$$

a function whose range is the complement of a system of $n+1$ radial segments of equal length, joining with equal angles at the origin. This conjecture has been proved [104], [25], [115] for functions $g \in \Sigma$ whose omitted set is starlike with respect to the origin, and also [77], [30], [33] for functions with $b_{j}=0$ for $1 \leqslant j \leqslant(n-1) / 2$. However, the general conjecture is false even for the third coefficient. As early as 1937, Bazilevič [8] had shown that among all odd functions $g \in \Sigma$, the sharp upper bound for $\left|b_{3}\right|$ is not $1 / 2$ as the conjecture asserts, but $1 / 2+e^{-6}$. The proof is a simple application of the Fekete-Szegö inequality (\$2) for $\left|a_{3}-\alpha a_{2}^{2}\right|$, with $\alpha=3 / 4$. In fact, the same argument gives (as Bazilevič observed) the sharp inequality

for functions

$$
\left|b_{2 m-1}\right| \leqslant 1 / m+(2 / m) e^{-2(m+1) /(m-1)}
$$

$$
g(z)=z+b_{m-1} z^{-m+1}+b_{2 m-1} z^{-2 m+1}+\cdots
$$

in $\Sigma$ with $m$-fold symmetry, $m \geqslant 2$. In particular, this disproves the conjecture $\left|b_{n}\right| \leqslant 2 /(n+1)$ for every odd index $n \geqslant 3$. 
Garabedian and Schiffer [47] used a variational method to extend the inequality $\left|b_{3}\right| \leqslant 1 / 2+e^{-6}$ to the full class $\Sigma$. Jenkins [78] and Bombieri [18] found alternate proofs, and Pommerenke [120] deduced the result from the Garabedian-Schiffer inequalities. Quite recently, Y. Kubota [81], [82] applied Jenkins' general coefficient theorem to show that for functions in $\Sigma$ with real coefficients, the sharp inequalities

$$
b_{5} \leqslant \frac{1}{3}+\frac{4}{507} \text { and } b_{4} \leqslant \frac{2}{5}+\frac{729}{163840} \text { for } b_{1} \geqslant 0
$$

hold. For $b_{1}<0$, the sharp upper bound of $b_{4}$ is slightly larger and is (presumably) no longer a rational number; Kubota [82] gives formulas for its calculation. In particular, this is the first published disproof of the conjecture $\left|b_{4}\right| \leqslant 2 / 5$.

These results demolish the conjecture $\left|b_{n}\right| \leqslant 2 /(n+1)$ and suggest that the coefficient problem for the class $\Sigma$ is considerably more difficult than the Bieberbach conjecture. To make matters worse, the conjecture $\left|b_{n}\right| \leqslant$ $2 /(n+1)$ is false asymptotically. Clunie [24] constructed functions $g \in \Sigma$ with $b_{n} \neq O\left(n^{-0.98}\right)$, and Pommerenke [117], [118], [120] gave examples for which $b_{n} \neq O\left(n^{-0.83}\right)$. On the positive side, Clunie and Pommerenke [27] improved the trivial estimate $b_{n}=O\left(n^{-1 / 2}\right)$ to $b_{n}=O\left(n^{-1 / 2-1 / 300}\right)$. The precise order of growth is not known.

11. Bounded univalent functions. Another problem of long standing is to find the sharp asymptotic estimate for the coefficients of bounded univalent functions. Each bounded function $f \in S$ maps the unit disk onto a region of finite area, and so its coefficients satisfy $\sum n\left|a_{n}\right|^{2}<\infty$. In particular, $a_{n}$ $=o\left(n^{-1 / 2}\right)$. This trivial estimate was thought to be essentially best possible until Clunie and Pommerenke [27], [120] improved it to $a_{n}=O\left(n^{-1 / 2-1 / 300}\right)$. Their work reveals a surprisingly close connection with the asymptotic problem for the class $\Sigma$.

In the opposite direction, Littlewood [89] had constructed examples of bounded univalent functions with $a_{n} \neq O\left(n^{-1}\right)$. Pommerenke [117] improved the result to $a_{n} \neq O\left(n^{-0.861}\right)$ and later [118] to $a_{n} \neq O\left(n^{-0.83}\right)$.

All of these constructions actually produce bounded univalent functions

$$
f(z)=z+a_{m+1} z^{m+1}+a_{2 m+1} z^{2 m+1}+\cdots
$$

with $m$-fold symmetry, where $m$ is an arbitrary positive integer. For such functions an old conjecture of Szegö asserts that $a_{n}=O\left(n^{2 / m-1}\right)$. This seems plausible because the coefficients of the $m$ th-root transform of the Koebe function, $f(z)=\left[k\left(z^{m}\right)\right]^{1 / m}$, have precisely this order of growth. The conjecture is of course true for $m=1$ and for $m=2$ (Littlewood-Paley [90]). Levin [86] proved it for $m=3$. He also showed for $m=4$ that $a_{n}=O\left(n^{-1 / 2} \log n\right)$, and for $m \geqslant 5$ that

$$
a_{n}=O\left(n^{-1 / 2}\{\log n\}^{1 / 2}\right)
$$


Littlewood's construction disproves Szegö's conjecture for all sufficiently large $m$, and Pommerenke's construction disproves it for all $m \geqslant 12$. For $4 \leqslant m$ $\leqslant 11$, the conjecture remains unsettled. For $m \geqslant 5$, Clunie and Pommerenke [27] improved Levin's result to $a_{n}=O\left(n^{-1 / 2-\varepsilon}\right)$ for some $\varepsilon>0$, and Pommerenke [120] showed that $\varepsilon$ can be taken to be $1 / 1600$.

These latter results do not actually require $m$-fold symmetry. The important condition is a growth estimate on $f$, which is achieved by requiring that the Taylor series be suitably lacunary.

12. Conclusion. The theory of univalent functions is an enormous subject with a literature extending back to the turn of the century. This brief survey could not possibly do it justice. I have been compelled to omit a number of interesting topics, or to mention them only in passing. Making no attempt at completeness, I have tried to convey the spirit of the subject and the flavor of recent developments, while recording some of the major unsolved problems. At the very least, the discussion should suggest that the venerable field of complex analysis is alive and well.

The reader who wishes to explore the subject in greater depth is advised to consult the recent book of Pommerenke [120] or my forthcoming book [38]. Some older sources are the books of Goluzin [55], Hayman [63], Jenkins [76], and Schaeffer and Spencer [132]. There are also recent books in Russian by Milin [102] and Lebedev [84].

I wisk to thank Albert Baernstein, Walter Hayman, and Glenn Schober for their helpful criticism of the first draft of this paper.

\section{REFERENCES}

1. D. Aharonov, Proof of the Bieberbach conjecture for a certain class of univalent functions, Israel J. Math. 8 (1970), 103-104. MR 42 \#486.

2. - Special topics in univalent functions, Lecture Notes, Univ. of Maryland, 1971.

3. Math. 15 (1973), 137-139. MR 48 \#520.

4. D. Aharonov and S. Friedland, On an inequality connected with the coefficient conjecture for functions of bounded boundary rotation, Ann. Acad. Sci. Fenn. Ser. AI No. 524 (1972), 14 pp. MR 48 \#519.

5. - On functions of bounded boundary rotation, Ann. Acad. Sci. Fenn. Ser. AI No. 585 (1974), $18 \mathrm{pp}$.

6. I. A. Aleksandrov and V. Ja. Gutljanskií, On the coefficient problem in the theory of univalent functions, Dokl. Akad. Nauk SSSR 188 (1969), 266-268 = Soviet Math. Dokl. 10 (1969), 1091-1094. MR 41 \#455.

7. A. Baernstein, Integral means, univalent functions and circular symmetrization, Acta Math. 133 (1974), 139-169.

8. I. E. Bazilevič, Supplement to the papers "Zum Koeffizientenproblem der schlichten Funktionen" and "Sur les théorèmes de Koebe-Bieberbach", Mat. Sb. 2 (44) (1937), 689-698. (Russian).

9. On distortion theorems and coefficients of univalent functions, Mat. Sb. (N.S.) 28 (70) (1951), 147-164. (Russian) MR 12, 600.

10. - A criterion for univalence of regular functions and for dispersion of their coefficients, Mat. Sb. (N.S.) 74 (116) (1967), 133-146 = Math. USSR-Sbornik 3 (1967), 123-137. MR 36 \#2790.

11. L. Bieberbach, Uber einige Extremalprobleme im Gebiete der konformen Abbildung, Math. Ann. 77 (1915-1916), 153-172. 
12. Uber die Koeffizienten derjenigen Potenzreihen, welche eine schlichte Abbildung des Einheitskreises vermitteln, S.-B. Preuss. Akad. Wiss. (1916), 940-955.

13. M. Biernacki, Sur les coefficients tayloriens des fonctions univalentes, Bull. Acad. Polon. Sci. Cl. III. 4 (1956), 5-8. MR 17, 957.

14. D. H. Bishouty, The Bieberbach conjecture for univalent functions with small second coefficients, Math. Z. 149 (1976), 183-187.

15. E. Bombieri, Sul problema di Bieberbach per le funzioni univalenti, Atti Accad. Naz. Lincei

Rend. Cl. Sci. Fis. Mat. Natur. (8) 35 (1963), 469-471. MR 29 \#3622.

16. - Sulla seconda variazione della funzione di Koebe, Boll. Un. Mat. Ital. (3) 22 (1967), 25-32. MR 35 \#6813.

17. - On the local maximum property of the Koebe function, Invent. Math. 4 (1967), 26-67. MR 36 \# 1635.

18. - A geometric approach to some coefficient inequalities for univalent functions, Ann. Scuola Norm. Sup. Pisa (3) 22 (1968), 377-397. MR 39 \#430.

19. D. A. Brannan, J. G. Clunie and W. E. Kirwan, On the coefficient problem for functions of bounded boundary rotation, Ann. Acad. Sci. Fenn. Ser. AI No. 523 (1973), 18 pp. MR 49 \#3108.

20. L. Brickman, Extreme points of the set of univalent functions, Bull. Amer. Math. Soc. 76 (1970), 372-374. MR 41 \#448.

21. L. Brickman, T. H. MacGregor and D. R. Wilken, Convex hulls of some classical families of univalent functions, Trans. Amer. Math. Soc. 156 (1971), 91-107. MR 43 \#494.

22. L. Brickman and D. Wilken, Support points of the set of univalent functions, Proc. Amer. Math. Soc. 42 (1974), 523-528. MR 48 \#6399.

23. Z. Charzynski and M. Schiffer, $A$ new proof of the Bieberbach conjecture for the fourth coefficient, Arch. Rational Mech. Anal. 5 (1960), 187-193. MR 22 \# 5746.

24. J. Clunie, On schlicht functions, Ann. of Math. (2) 69 (1959), 511-519. MR 21 \#6438.

25. , On meromorphic schlicht functions, J. London Math. Soc. 34 (1959), 215-216. MR 21 \# 5737.

26. J. Clunie and P. L. Duren, Addendum: An arclength problem for close-to-convex functions, J. London Math. Soc. 41 (1966), 181-182. MR 32 \#7725.

27. J. Clunie and $\mathrm{Ch}$. Pommerenke, On the coefficients of univalent functions, Michigan Math. J. 14 (1967), 71-78. MR 34 \# 7786.

28. J. Dieudonné, Sur les fonctions univalentes, C. R. Acad. Sci. Paris 192 (1931), 1148-1150.

29. N. Dunford and J. T. Schwartz, Linear operators, Vol. I, Interscience, New York, 1958. MR 22 \# 8302.

30. P. L. Duren, Coefficient estimates for univalent functions, Proc. Amer. Math. Soc. 13 (1962), 168-169. MR 28 \# 1286.

31. - An arclength problem for close-to-convex functions, J. London Math. Soc. 39 (1964), 757-761. MR 30 \#241.

32. - Theory of $H^{p}$ spaces, Academic Press, New York, 1970. MR 42 \# 3552.

33. _ Coefficients of meromorphic schlicht functions, Proc. Amer. Math. Soc. 28 (1971), 169-172. MR 42 \#6212.

34. - Estimation of coefficients of univalent functions by a Tauberian remainder theorem, J. London Math. Soc. (2) 8 (1974), 279-282. MR 51 \# 877.

35. - Asymptotic behavior of coefficients of univalent functions, Advances in Complex Function Theory (Maryland 1973/74), Lecture Notes in Math., vol. 505, Springer-Verlag, Berlin and New York, 1976, pp. 17-23.

36. - Applications of the Garabedian-Schiffer inequality, J. Analyse Math. 30 (1976), 141-149.

37. Subordination, Sympos. on Complex Analysis (Kentucky 1976), Lecture Notes in Math., vol. 599, Springer-Verlag, Berlin and New York, 1977, pp. 22-29.

38. __ Univalent functions, Springer-Verlag (to appear).

39. P. L. Duren and M. Schiffer, The theory of the second variation in extremum problems for univalent functions, J. Analyse Math. 10 (1962/63), 193-252. MR 27 \#284.

40. G. Ehrig, The Bieberbach conjecture for univalent functions with restricted second coefficients, J. London Math. Soc. (2) 8 (1974), 355-360.

41. - Coefficient estimates concerning the Bieberbach conjecture, Math. Z. 140 (1974), 111-126. 
42. M. Fekete and G. Szegö, Eine Bemerkung über ungerade schlichte Funktionen, J. London Math. Soc. 8 (1933), 85-89.

43. J. Feng and T. H. MacGregor, Estimates on integral means of the derivatives of univalent functions, J. Analyse Math. 29 (1976), 203-231.

44. C. H. FitzGerald, Quadratic inequalities and coefficient estimates for schlicht functions, Arch. Rational Mech. Anal. 46 (1972), 356-368. MR 49 \#557.

45. S. Friedland, On a conjecture of Robertson, Arch. Rational Mech. Anal. 37 (1970), 255-261. MR 41 \#456.

46. P. R. Garabedian and M. Schiffer, $A$ proof of the Bieberbach conjecture for the fourth coefficient, J. Rational Mech. Anal. 4 (1955), 427-465. MR 17, 24.

47. - A coefficient inequality for schlicht functions, Ann. of Math. (2) 61 (1955), 116-136. MR 16, 579.

48. - The local maximum theorem for the coefficients of univalent functions, Arch. Rational Mech. Anal. 26 (1967), 1-32. MR 37 \# 1584.

49. P. R. Garabedian, G. G. Ross and M. Schiffer, On the Bieberbach conjecture for even $n, J$. Math. Mech. 14 (1965), 975-989. MR 32 \#207.

50. G. M. Goluzin, Some bounds on the coefficients of univalent functions, Mat. Sb. 3 (45) (1938), 321-330. (Russian).

51. - On distortion theorems and coefficients of univalent functions, Mat. Sb. N. S. 19 (61) (1946), 183-202. (Russian). MR 8, 325.

52. - On distortion theorems and the coefficients of univalent functions, Mat. Sb. N. S. 23 (65) (1948), 353-360. (Russian). MR 10, 602.

53. - Some questions of the theory of univalent functions, Trudy Mat. Inst. Steklov. 27 (1949), 1-112. (Russian). MR 13, 123.

54. —, On typically real functions, Mat. Sb. N. S. 27 (69) (1950), 201-218. (Russian). MR $12,490$.

55. - Geometric theory of functions of a complex variable, GITTL, Moscow, 1952; German transl., VEB Deutscher Verlag, Berlin, 1957; 2nd ed., "Nauka", Moscow, 1966; English transl., Transl. Math. Monographs, vol. 26, Amer. Math. Soc., Providence, R.I., 1969. MR 15, 112; 19, 735; 36 \# 2793; 40 \#308.

56. A. W. Goodman, Open problems on univalent and multivalent functions, Bull. Amer. Math. Soc. 74 (1968), 1035-1050. MR 38 \#315.

57. — The valence of sums and products, Canad. J. Math. 20 (1968), 1173-1177. MR 38 \#314.

58. G. S. Goodman, On the determination of univalent functions with prescribed initial coeffcients, Arch. Rational Mech. Anal. 24 (1967), 78-81. MR 34 \#4478.

59. A. Z. Grinšpan, Logarithmic coefficients of functions in the class $S$, Sibirsk. Mat. Ž. 13 (1972), 1145-1157, 1199 = Siberian Math. J. 13 (1972), 793-801. MR 48 \#6403.

60. T. H. Gronwall, Some remarks on conformal representation, Ann. of Math. 16 (1914-1915), 72-76.

61. H. Grunsky, Koeffizientenbedingungen für schlicht abbildende meromorphe Funktionen, Math. Z. 45 (1939), 29-61.

62. W. K. Hayman, The asymptotic behaviour of p-valent functions, Proc. London Math. Soc.

(3) 5 (1955), 257-284. MR 17, 142.

63. - Multivalent functions, Cambridge Univ. Press, Cambridge, 1958. MR 21 \# 7302.

64. - Bounds for the large coefficients of univalent functions, Ann. Acad. Sci. Fenn. Ser. AI No. 250 (1958), 13pp. MR 20 \#3292.

65. On successive coefficients of univalent functions, J. London Math. Soc. 38 (1963), 228-243. MR 26 \#6382.

66. Coefficient problems for univalent functions and related function classes, J. London Math. Soc. 40 (1965), 385-406. MR 31 \#3592.

67. W. Hengartner and G. Schober, Extreme points for some classes of univalent functions, Trans. Amer. Math. Soc. 185 (1973), 265-270. MR 49 \#559.

68. D. Horowitz, A refinement for coefficient estimates of univalent functions, Proc. Amer. Math. Soc. 54 (1976), 176-178.

69. - Coefficient estimates for univalent polynomials, J. Analyse Math. (to appear).

70. J. A. Hummel, Bounds for the coefficient body of univalent functions, Arch. Rational Mech. Anal. 36 (1970), 128-134. MR 42 \#6213. 
71. Lectures on variational methods in the theory of univalent functions, Lecture Notes, Univ. of Maryland, 1972.

72. L. P. Il'ina, On the mutual growth of neighboring coefficients of univalent functions, Mat. Zametki 4 (1968), 715-722 = Math. Notes 4 (1968), 918-922. MR 39 \# 1644.

73. Estimates for the coefficients of univalent functions in terms of the second coefficient, Mat. Zametki 13 (1973), 351-357 = Math. Notes 13 (1973), 215-218. MR 47 \# 7019.

74. J. A. Jenkins, On a problem of Gronwall, Ann. of Math. (2) 59 (1954), 490-504. MR 15, 786.

75. - A general coefficient theorem, Trans. Amer. Math. Soc. 77 (1954), 262-280. MR 16, 232.

76. - 'Univalent functions and conformal mapping, Springer-Verlag, Berlin, 1958. MR 20 \#3288.

77. On certain coefficients of univalent functions. II, Trans. Amer. Math. Soc. 96 (1960), 534-545. MR 23 \# A309.

78. - On certain coefficients of univalent functions, Analytic Functions, Princeton Univ.

Press, Princeton, N.J., 1960, pp. 159-194. MR 22 \# 8126a.

79. - Some area theorems and a special coefficient theorem, Illinois J. Math. 8 (1964), 80-99. MR 28 \#4097.

80. W. Kaplan, Close-to-convex schlicht functions, Michigan Math. J. 1 (1952), 169-185 (1953). MR 14, 966.

81. Yoshihisa Kubota, A coefficient inequality for certain meromorphic univalent functions, Kōdai Math. Sem. Rep. 26 (1974/75), 85-94. MR 51 \#5915.

82. On the fourth coefficient of meromorphic univalent functions, Ködai Math. Sem. Rep. 26 (1974/75), 267-288. MR 52 \#732.

83. Kung Sun, Contributions to the theory of schlicht functions. II: The coefficient problem, Sci. Sinica 4 (1955), 359-373.

84. N. A. Lebedev, The area principle in the theory of univalent functions, "Nauka", Moscow, 1975. (Russian).

85. G. B. Leeman, The seventh coefficient of odd symmetric univalent functions, Duke Math. J. 43 (1976), 301-307.

86. V. I. Levin, Ein Beitrag zum Koeffizientenproblem der schlichten Funktionen, Math. Z. 38 (1933), 306-311.

87. - Some remarks on the coefficients of schlicht functions, Proc. London Math. Soc. (2) 39 (1935), 467-480.

88. J. E. Littlewood, On inequalities in the theory of functions, Proc. London Math. Soc. 23 (1925), 481-519.

89. - On the coefficients of schlicht functions, Quart. J. Math. Oxford Ser. 9(1938), $14-20$.

90. J. E. Littlewood and R. E. A. C. Paley, A proof that an odd schlicht function has bounded coefficients, J. London Math. Soc. 7 (1932), 167-169.

91. C. Loewner (K. Löwner), Untersuchungen über die Verzerrung bei konformen Abbildungen des Einheitskreises $|z|<1$, die durch Funktionen mit nicht verschwindender Ableitung geliefert werden, Ber. Verh. Sächs. Ges. Wiss. Leipzig 69 (1917), 89-106.

92. - Untersuchungen über schlichte konforme Abbildungen des Einheitskreises. I, Math. Ann. 89 (1923), 103-121.

93. A. J. Lohwater, G. Piranian and W. Rudin, The derivative of a schlicht function, Math. Scand. 3 (1955), 103-106. MR 17, 249.

94. K. W. Lucas, On successive coefficients of areally mean p-valent functions, J. London Math. Soc. 44 (1969), 631-642. MR 39 \#4379.

95. T. H. MacGregor, Applications of extreme-point theory to univalent functions, Michigan Math. J. 19 (1972), 361-376. MR 47 \#447.

96. F. Marty, Sur le module des coefficients de Maclaurin d'une fonction univalente, C. R. Acad. Sci. Paris 198 (1934), 1569-1571.

97. I. M. Milin, The area method in the theory of univalent functions, Dokl. Akad. Nauk SSSR 154 (1964), 264-267 = Soviet Math. Dokl. 5 (1964), 78-81. MR 28 \# 1283.

98. - Estimation of coefficients of univalent functions, Dokl. Akad. Nauk SSSR 160 (1965), 769-771 = Soviet Math. Dokl. 6 (1965), 196-198. MR 30 \# 3206.

99. - On the coefficients of univalent functions, Dokl. Akad. Nauk SSSR 176 (1967), 1015-1018 = Soviet Math. Dokl. 8 (1967), 1255-1258. MR 36 \#5328. 
100. - Adjacent coefficients of univalent functions, Dokl. Akad. Nauk SSSR 180 (1968), 1294-1297 = Soviet Math. Dokl. 9 (1968), 762-765. MR 38 \#318.

101. Hayman's regularity theorem for the coefficients of univalent functions, Dokl. Akad. Nauk SSSR 192 (1970), 738-741 = Soviet Math. Dokl. 11 (1970), 724-728. MR 42 \#3269.

102. - Univalent functions and orthonormal systems, "Nauka", Moscow, 1971 (Russian). MR 51 \# 5916.

103. Z. Nehari, On the coefficients of univalent functions, Proc. Amer. Math. Soc. 8 (1957), 291-293. MR 18, 728.

104. Z. Nehari and E. Netanyahu, On the coefficients of meromorphic schlicht functions, Proc. Amer. Math. Soc. 8 (1957), 15-23. MR 18, 648.

105. E. Netanyahu, On univalent functions in the unit disk whose image contains a given disk, J. Analyse Math. 23 (1970), 305-322. MR 43 \#6420.

106. R. Nevanlinna, Uber die konforme Abbildung von Sterngebieten, Översikt av Finska Vetenskaps-Soc. Förh. 63(A) (1920-21), no. 6, 1-21.

107. A. E. Obrock, An inequality for certain schlicht functions, Proc. Amer. Math. Soc. 17 (1966), 1250-1253. MR 34 \#6075.

108. M. Ozawa, On the Bieberbach conjecture for the sixth coefficient, Kōdai Math. Sem. Rep. 21 (1969), 97-128. MR 39 \# 432.

109. An elementary proof of the Bieberbach conjecture for the sixth coefficient, Kōdai Math. Sem. Rep. 21 (1969), 129-132. MR 40 \#333.

110. R. N. Pederson, $A$ proof of the Bieberbach conjecture for the sixth coefficient, Arch. Rational Mech. Anal. 31 (1968/69), 331-351. MR 39 \#431.

111. - A note on the local coefficient problem, Proc. Amer. Math. Soc. 20 (1969), 345-347. MR 38 \# 4667.

112. R. Pederson and M. Schiffer, A proof of the Bieberbach conjecture for the fifth coefficient, Arch. Rational Mech. Anal. 45 (1972), 161-193. MR 47 \#453.

113. A. Pfluger, Lineare Extremalprobleme bei schlichten Funktionen, Ann. Acad. Sci. Fenn. Ser. A I No. 489 (1971), 32 pp. MR 45 \#5337.

114. G. Pólya and I. J. Schoenberg, Remarks on de la Vallée Poussin means and convex conformal maps of the circle, Pacific J. Math. 8 (1958), 295-334. MR 20 \#7181.

115. Ch. Pommerenke, On meromorphic starlike functions, Pacific J. Math. 13 (1963), 221-235. MR 27 \#280.

116. - Uber die Faberschen Polynome schlichter Funktionen, Math. Z. 85 (1964), 197-208.

MR 29 \#6028.

117. - On the coefficients of univalent functions, J. London Math. Soc. 42 (1967), 471-474. MR 36 \#3329.

118. - Relations between the coefficients of a univalent function, Invent. Math. 3 (1967), 1-15. MR 36 \#3972.

119. - On a variational method for univalent functions, Michigan Math. J. 17 (1970), 1-3. MR 41 \#452.

120. - Univalent functions (with a chapter on quadratic differentials by G. Jensen), Vandenhoeck and Ruprecht, Göttingen, 1975.

121. I. I. Privalov, On functions giving a univalent conformal mapping, Mat. Sb. 31 (1924), 350-365. (Russian).

122. M. O. Reade, On close-to-convex univalent functions, Michigan Math. J. 3 (1955), 59-62. MR 17, 25.

123. M. S. Robertson, A remark on the odd schlicht functions, Bull. Amer. Math. Soc. 42 (1936), 366-370.

124. The generalized Bieberbach conjecture for subordinate functions, Michigan Math.

J. 12 (1965), 421-429. MR 32 \#2576.

125. Q Quasi-subordination and coefficient conjectures, Bull. Amer. Math. Soc. 76 (1970), 1-9. MR 40 \#4441.

126. - Quasi-subordinate functions, Mathematical Essays Dedicated to A. J. Macintyre, Ohio Univ. Press, Athens, Ohio, 1970, pp. 311-330. MR 42 \#7885.

127. W. Rogosinski, Über positive harmonische Entwicklungen und typisch-reelle Potenzreihen, Math. Z. 35 (1932), 93-121.

128. On subordinate functions, Proc. Cambridge Philos. Soc. 35 (1939), 1-26. 
129. , On the coefficients of subordinate functions, Proc. London Math. Soc. (2) 48 (1943), 48-82. MR 5, 36.

130. St. Ruscheweyh and T. Sheil-Small, Hadamard products of schlicht functions and the PólyaSchoenberg conjecture, Comment. Math. Helv. 48 (1973), 119-135. MR 48 \#6393.

131. A. C. Schaeffer and D. C. Spencer, The coefficients of schlicht functions, Duke Math. J. 10 (1943), 611-635.

132. __ Coefficient regions for schlicht functions, Amer. Math. Soc. Colloq. Publ., vol. 35, Amer. Math. Soc., Providence, R.I., 1950. MR 12, 326.

133. M. Schiffer, Sur un problème d'extrémum de la représentation conforme, Bull. Soc. Math. France 66 (1938), 48-55.

134. $A$ method of variation within the family of simple functions, Proc London Math. Soc. (2) 44 (1938), $432-449$.

135. - On the coefficients of simple functions, Proc. London Math. Soc. (2) 44 (1938), $450-452$.

136. - Variation of the Green function and theory of the p-valued functions, Amer. J. Math. 65 (1943), 341-360. MR 4, 215.

137. - Some recent developments in the theory of conformal mapping, Appendix to $\mathbf{R}$. Courant, Dirichlet's Principle, Conformal Mapping, and Minimal Surfaces, Interscience, New York, 1950. MR 12, 90.

138. - Applications of variational methods in the theory of conformal mapping, Proc. Sympos. Appl. Math., vol. 8, Amer. Math. Soc., Providence, R.I., 1958, pp. 93-113. MR 20 \# 965.

139. - Univalent functions whose $n$ first coefficients are real, J. Analyse Math. 18 (1967), 329-349. MR 35 \# 5603.

140. - On the coefficient problem for univalent functions, Trans. Amer. Math. Soc. 134 (1968), 95-101. MR 37 \#249.

141. G. Schober, Univalent functions-selected topics, Lecture Notes in Math., vol. 478, Springer-Verlag, Berlin-New York, 1975.

142. I. Schur, Bemerkungen zur Theorie der beschränkten Bilinearformen mit unendlich vielen Veränderlichen, J. Reine Angew. Math. 140 (1911), 1-28.

143. T. Sheil-Small, On the convolution of analytic functions, J. Reine Angew. Math. 258 (1973), 137-152. MR 47 \#9295.

144. N. A. Sirokov, Hayman's regularity theorem, Zap. Naučn. Sem. Leningrad. Otdel. Mat. Inst. Steklov. (LOMI) 24 (1972), 182-200. (Russian). MR 46 \# 3774.

145. O. Szász, Uber Funktionen, die den Einheitskreis schlicht abbilden, Jber. Deutsch. Math.Verein. 42 (1933), 73-75.

Department of Mathematics, University of Michigan, AnN Arbor, Michigan 48109 